\title{
The interaction between the dopaminergic and the serotonergic systems in the 6-OHDA rat model of Parkinson's disease
}

\author{
Daphna Sourani", Gadi Goelman \\ MRI/MRS Laboratory, The Human Biology Research Center, Hadassah Hebrew University Medical Center, Jerusalem, Israel; \\ *Corresponding Author: dafi.sourani@gmail.com
}

Received 3 September 2012; revised 12 October 2012; accepted 21 October 2012

\begin{abstract}
This review summarizes published results that are related to the coupling between the dopaminergic and the serotonergic systems and their association to Parkinson's disease. We focus on the 6-hydroxydopamine rat model of Parkinson's disease to better understand how dopamine dysfunction affects the serotonergic system, and furthermore to investigate whether a bidirectional coupling exists and how it affects functionality and behavior. The accumulated evidence supports a proposed mechanism for this coupling that evolves the lateral habenula.
\end{abstract}

Keywords: Parkinson's Disease; Depression; 6-Hydroxydopamine; Lateral Habenula;

Manganese-Enhanced-MRI

\section{INTRODUCTION}

Parkinson's Disease (PD) is a neurodegenerative disease which is primarily known by its movement disorders that are believed to be associated with the degeneration of dopamine (DA) neurons in the substantia nigra pars compacta (SNc). In addition to motor symptoms, a large percentage of PD patients suffer from depression and various cognitive impairments that in many cases even precede the motor symptoms [1,2]. These symptoms are believed to be associated with the serotonergic (5-HT) system, which raises the issue of the coupling between these systems.

Several studies in PD human patients have reported evidence of coupling between the dopaminergic and the serotonergic systems. For example, lower levels of serotonin and reduced 5-HT1A receptor binding potential in the raphe nuclei have been found $[3,4]$. Other studies have indicated reduced levels of 5-hydroxyindoleacetic acid (5-HIAA), a breakdown product of 5-HT, in the cerebrospinal fluid of PD patients, and an additional reduction of 5-HIAA in depressed PD patients [5,6]. Reduced dopamine levels $[7,8]$ and increased D2 dopamine receptor-density in the basal ganglia (BG) and the cerebellum [9] were found in depressed patients whereas serotonergic stimulation in the prefrontal cortex [10] or the striatum [11] led to dopamine release. Furthermore, post mortem studies performed on PD human brains suggest that serotonergic cell loss in the Raphe nuclei in some cases may occur earlier than DA cell death $[12,13]$. Anatomical findings confirm the relationship between these systems. Serotonin-containing cell bodies of the raphe nuclei send projections to dopaminergic cells both in the VTA and the SN, and to their terminal fields in the nucleus accumbens, prefrontal cortex, and striatum [1419].

All the above suggest that there is a coupling between the dopaminergic and the serotonergic systems in the sense that an alteration in one system affects the functionality of the other. Understanding this coupling and its physiological mechanisms is essential for the treatment of the non-motor symptoms of PD in particular.

This review focuses on studies, performed on a rat model of PD, that demonstrate this coupling. The 6-hydroxydopamine rat model of PD will be introduced and studies related to coupling between the dopaminergic and the serotonergic systems will be described in order to better understand the mechanism underlying this coupling

\section{MATERIALS AND METHODS}

\subsection{The 6-OHDA Rat PD Model and Its Validity for Motor and Non-Motor Symptoms in PD}

6-OHDA is a neurotoxin whose direct injection into the brain causes dopamine cell death; thus, it mimics SNc neuronal loss in PD [20]. Since 6-OHDA does not efficiently pass the blood-brain barrier, the compound is 
injected directly into the brain. The most widely used injection is unilateral, since rats with bilateral lesions require intensive nursing care [21]. The injection may be targeted at any point along the nigrostriatal path, although the amount of degeneration and the subsequent behavioral symptoms differ [22].

The 6-OHDA rat model has contributed to basic brain research on DA neurons and their interactions, as well as to a better understanding of Parkinson's disease. It has also served as an experimental foundation to develop new drugs and treatment strategies, including modes of drug delivery and surgical approaches $[23,24]$. However, the model has a number of caveats. First, the model is involved with unilateral and not bilateral dopamine cell loss. This makes the model differ from human PD since in most cases both hemispheres are damaged. It could be however, thought as mimicking some cases of earlier stages of the disease in which one hemisphere is damaged.

Second, the 6-OHDA model does not include the Lewy bodies whereas in human, idiopathic PD is characterized by the development of Lewy neurites (LNs) and Lewy bodies (LBs). Moreover, they begin to evolve outside the SNc (for example in the olfactory bulb or the raphe nuclei) before evolving in the SNc. As such, LNs and LBs can be found to a mild or moderate extent before the appearance of motor dysfunctions [25-33]. Thus, the 6-OHDA is not suitable for clarifying the mechanisms leading to the degeneration of DA neurons.

Third, in the 6-OHDA model, the lesion is made in the $\mathrm{SNc}$ alone (or in other locations along the nigrostriatal route). By contrast in human $\mathrm{PD}$, there are "extra nigral” findings, such as the LB described above, which have prompted hypotheses that PD may not be of dopaminergic source. For example, Hawkes and Braak [34] suggested that PD could be caused by infection in which a viral neurotropic agent enters the brain via the nasal (anterograde transmission to temporal lobe) and/or gastric (retrograde transmission to medulla via the Meissner's plexus and vagal neurones) routes. Thus, the 6-OHDA model cannot be used to study the mechanism that generate PD but is more suitable to explore the effects of dopamine cell death on other neuronal systems and on behavior.

In light of these limitation, the question that should be raised is whether the 6-OHDA model is an appropriate model to characterize mood disorders and specifically depression in PD.

Although assessment of mood-related behaviors in animals is complex and can have multiple interpretations $[35,36]$, several behavioral tests are now standard and are used to define facets of "depressive-like" behavior. For example, the forced swimming test and the sucrose preference test are used to assess an anhedonic-depressive- like effect. The novelty suppressed feeding test and the elevated plus maze are used to assess anxiogenic-like activity. Several studies have found depressive-like behavior in 6-OHDA injected rats on the basis of such tests. For example, Tadaiesky et al. used rats with 6-OHDA bilateral injection to the striatum, which causes a gradually cell loss in the SNc following the injection [37]. This lesion mimics more closely the slowly evolving nature of the nigral lesion of PD and creates a window on the early phases of PD. In this study, decreased sucrose consumption was found in the 6-OHDA lesioned rats, as well as increased immobility time on the forced swimming test. Moreover, anxiogenic-like activity in the elevated plus maze was found [38].

Similar findings were observed by Branchi et al. [39] who detected increased immobility time on the forced swimming test and a decrease in anxiety behavior in the elevated plus maze in rats with bilateral 6-OHDA lesions to the striatum. Sourani et al. reported increased immobility time on the forced swimming test, as well as increased anxiety behavior on the novelty suppressed feeding test in rats subjected to a unilateral 6-OHDA lesion to the SNc [40].

Winter et al. used rats with a unilateral 6-OHDA lesion to the VTA and SNc with different extents of lesions. Using a learned helplessness inescapable shock paradigm, they showed that lesions of DAergic nigral cells induced depressive behavior that increased with the extent of lesion of either the SNc or the VTA [41].

Santiago et al. tested rats with a bilateral 6-OHDA lesion to the SNc. In the 6-OHDA group compared to the sham group, increased immobility time on the forced swimming test was found, as well as decreased sucrose consumption [42].

All these results document different behavioral disturbances in various 6-OHDA rat models that are in line with findings in human PD patients; they hence confirm the relevance of these models to the study of mood disorders in PD.

Of a key interest is the fact that these behavioral changes take place without any serotonin cell loss. Furthermore, the DA lesion is not preceded by raphe or other cell death, and no LB or LN are involved. In addition, the motor symptoms in the 6-OHDA models are clearly not preceded by behavioral dysfunction. Therefore, it makes sense to assume that the alterations in the dopaminergic system cause alterations in the functionality of the serotonergic system. This coupling is discussed in the next section.

\subsection{Physiological Evidence for the Coupling between the Dopaminergic and the Serotonergic Systems}

Several studies have reported enhanced 5-HT neuronal 
firing rates in the raphe nuclei of 6-OHDA injected rats. Using extra cellular recordings, Wang et al. showed an increase in the mean firing rate of the dorsal and median raphe nuclei as well as a decreased neuronal volume, and a change of the firing pattern toward a more bursty one in rats with a unilateral 6-OHDA lesion of the SNc. Moreover, a decreased response of these neurons to 5-HT $1 \mathrm{~A}$ receptor was shown, reflecting a dysfunction of this receptor in 6-OHDA lesioned rats [43]. Kaya et al. confirmed this by showing an increased firing rate of 5-HT neurons of the dorsal raphe in rats with 6-OHDA injection to the lateral ventricle [44].

Temel et al. used extra cellular single unit recordings to show that bilateral STN high frequency stimulation inhibits the high activity of the dorsal raphe 5-HT neurons in 6-OHDA injected rats to the lateral ventricle. These rats also showed a depressive like behavior on the forced swimming test [45].

Sourani et al. also shown that chronic apomorphine treatment, a dopamine replacement treatment, of rats with a unilateral 6-OHDA lesion of the SNc improves behavior as measured with the forced swimming and novelty suppressed feeding tests [40].

These results demonstrate the existence of active bidirectional coupling between the dopaminergic and the serotonergic (and specifically the raphe neclei) systems. Nevertheless it remains unclear how these systems are coupled and the mechanism behind this coupling. Studies that attempt to respond to this question are discussed below.

\subsection{Possible Mechanism for the Coupling between the Dopaminergic and the Serotonergic Systems}

Pelled et al. [46] used MRI with an intracranial manganese $(\mathrm{Mn}+2)$ injection, a method known as Manganese Enhanced MRI (MEMRI) [47-49] to better understand this coupling. In MEMRI, $M+2$ ions are injected into a pre-defined brain region. Manganese ions are chemically and physically similar to calcium ions and are therefore assumed to enter the neurons through voltage gated calcium channels, and then move in an anterograde manner along axons and across synapses [47]. As manganese is paramagnetic, its presence causes a signal enhancement in T1-weighted images, which is approximately proportional to its concentration [47]. Based on these characteristics of $\mathrm{Mn}+2$, the MEMRI method provides spatiotemporal information on the efferent connectivity of a pre-defined site. Pelled et al. found enhanced neuronal efferent connectivity of the BG output nuclei, particularly the globus palidus internal (GPi) to the lateral habenula (LHb), and enhanced efferent connectivity of the LHb particularly to the dorsal raphe nuclei (DRN) in unilateral 6-OHDA injected rats. Based on these findings, Pelled et al. suggested that the linkage between the dopaminergic and the serotonergic systems takes place through the habenula.

Pelled et al.'s claim has some anatomical support. Studies have shown that the medial division of the LHb receives afferents primarily from limbic brain regions, whereas its lateral division is mainly innervated by the BG. Furthermore, LHb efferents primarily target nuclei containing monoaminergic neurons, thus exerting control over these systems [50-52]. Furthermore, there are studies that demonstrate the involvement of the LHb in depression. Sartorius et al. reported deep brain stimulation to the LHb in a treatment-resistant depressed patient that resulted in full remission [53,54]. Other studies have reported hyperactivity of the LHb in depressed humans $[55,56]$ and in certain animal models of depression [57, 58].

Based on the above hypothesis and findings, a study by Sourani et al. [40] hypothesized that enhanced LHb excitability is a direct consequence of 1) the enhanced GPi excitability known to occur in PD and 2) the excitatory nature of the GPi $\rightarrow$ LHb projection [59]. Since the $\mathrm{LHb} \rightarrow$ DRN projection is effectively inhibitory [60], it suggests a down-regulation of the serotonergic system in 6-OHDA injected rats that might account for depression. They further hypothesized that due to the central role of the LHb, functional suppression of its activity would uncouple the serotonergic from the dopaminergic system and therefore would help alleviate depressive behavior. In their study they used a unilateral 6-OHDA rat model which was also validated as appropriate for the study of depression using behavioral tests. Using the same MEMRI method that was described earlier but with a $\mathrm{Mn}+2$ injection to the raphe nuclei, as well as behavioral tests, they showed reduced raphe excitability in 6-OHDA injected rats compared to sham injected rats. Together with the results of Pelled et al., these findings suggest a sequence of events starting with dopamine cell loss and continuing with enhanced LHb efferent connectivity, reduced raphe excitability and depression-like behavior.

As described earlier, several studies have reported enhanced 5-HT neuronal firing rates in the raphe nuclei of 6-OHDA unilaterally-injected rats [43-45] presumably contradicting Sourani et al.'s results. However, a decreased neuronal volume of raphe neurons in 6-OHDA unilaterally injected rats were reported [43]. Since the effective connectivity as measured by the MEMRI method is a complex weight of the number of active neurons and their firing efficiency, it is expected to be reduced with decreased neuronal volume as indeed was observed by Sourani et al.

Furthermore, Sourani et al. showed that apomorphine, which is a dopaminergic agonist [61,62] and is also 
thought to reduce habenula activity [63], partially normalized raphe connectivity and depressive-like behavior. These results strengthen the claim for the critical role of the habenula in modulating raphe excitability and connectivity and therefore behavior.

Finally they reported the unambiguous finding that depressive behavior can be improved by functional suppression of habenula activity. These results directly demonstrate the role of the LHb in the coupling between the dopaminergic and the serotonergic systems, and are consistent with a report on a human depressed patient [54].

\section{CONCLUSIONS}

Based on the postulate that PD is primarily associated with dopamine cell death in the SNc, the 6-OHDA injection rat model has been used in many studies to better understand the disease, particularly in relation to its motor and non-motor symptoms. Since the lesion in this model is specifically to DA neurons in the nigrostriatal route with no direct involvement of other structures and the model does not generate LB or LN, it can be used to study the coupling between the dopaminergic system and other systems. A lesion of DA cells is expected to have a direct effect on other systems through the coupling which could develop into a mutual influence affecting bidirectional connectivity. In fact several studies involving the 6-OHDA model have shown a coupling between the dopaminergic and the serotonergic systems. These include changes in the firing pattern of the raphe 5-HT neurons in 6-OHDA lesioned rats $[43,44]$, inhibition in the activity of raphe 5-HT neurons following STN high frequency stimulation of 6-OHDA lesioned rats [45]. Furthermore, the behavior of 6-OHDA injected rats was shown to be depressive-like on a variety of behavioral tests, such as the sucrose preference test, the forced swimming test, the novelty suppressed feeding test, etc. [38-42], confirming the validity of the model to the study of motor and non motor symptoms.

It is not clear, however, what constitutes the mechanisms behind the coupling between the dopamine and other systems. Of major interest is the coupling between the dopaminergic and the seretonergic systems since it could account for the high percentage of PD patients who suffer from clinically identified depression and other deficits related to the serotonin system. We have put forward a comparatively simple (perhaps over-simplified) mechanism for this coupling [46,40]. It evolves as a cascade of events that results from DA cell death. It includes enhanced LHb excitability, down-regulation of raphe activity, reduced serotonin production and altered raphe connectivity. All these have been suggested to cause depression. This hypothesis was based on behav- ioral results performed with and without dopamine replacement therapy and with and without bilateral lesion of the LHb. This latter finding was consistent with the success of a DBS procedure that was performed on a treatment resistant depressive patient [54]. On the other hand it was based on direct connectivity measurements that estimated the effective connectivity of the BG output nuclei, the LHb and the raphe nuclei using the manganese enhanced MRI method.

Clearly further studies are needed to better understand the coupling between the systems, and will pave the way to new therapeutic approaches to assist PD patients.

\section{REFERENCES}

[1] Nilsson, F.M., Kessing, L.V. and Bolwig, T.G. (2001) Increased risk of developing Parkinson's disease for patients with major affective disorder: A register study. Acta Psychiatrica Scandinavica, 104, 380-386. doi:10.1034/j.1600-0447.2001.00372.x

[2] Schurman, A.G., van den Akker, M., Ensinck, K.T., Metsemakers, J.F., Knottnerus, J.A., Leentjens, A.F. and Buntinx, F. (2002) Increased risk of Parkinson's disease after depression: A retrospective cohort study. Neurology, 58, 1501-1504. doi:10.1212/WNL.58.10.1501

[3] Doder, M., Rabiner, E.A., Turjanski, N., Lees, A.J. and Brooks, D.J. (2003) Tremor in Parkinson's disease and serotonergic dysfunction: An 11C-WAY 100635 PET study. Neurology, 60, 601-605.

doi:10.1212/01.WNL.0000031424.51127.2B

[4] Guttman, M., Boileau, I., Warsh, J., Saint-Cyr, J.A., Ginovart, N., McCluskey, T., Houle, S., Wilson, A., Mundo, E., Rusjan, P., Meyer, J. and Kish, S.J. (2007) Brain serotonin transporter binding in non-depressed patients with Parkinson's disease. European Journal of Neurology, 14, 523-528. doi:10.1111/j.1468-1331.2007.01727.x

[5] Mayeux, R., Stern, Y., Cote, L. and Williams, J.B. (1984) Altered serotonin metabolism in depressed patients with Parkinson's disease. Neurology, 34, 642-646. doi:10.1212/WNL.34.5.642

[6] Chen, C.P., et al. (1998) Post-synaptic 5-HT1A and 5HT2A receptors are increased in Parkinson's disease neocortex. Annals of the New York Academy of Sciences, 861, 288-289. doi:10.1111/j.1749-6632.1998.tb10229.x

[7] Lambert, G., Johansson, M., Agren, H. and Friberg, P. (2000) Reduced brain norepinephrine and dopamine release in treatment-refractory depressive illness: Evidence in support of the catecholamine hypothesis of mood disorders. Archives of General Psychiatry, 57, 787-793. doi:10.1001/archpsyc.57.8.787

[8] McLean, A., Rubinsztein, J.S., Robbins, T.W. and Sahakian, B.J. (2004) The effects of tyrosine depletion in normal healthy volunteers: implications for unipolar depression. Psychopharmacology (Berl), 171, 286-297. doi:10.1007/s00213-003-1586-8

[9] D’Haenen, H.A. and Bossuyt, A. (1994) Dopamine D2 receptors in depression measured with single photon 
emission computed tomography. Biological Psychiatry, 35, 128-132. doi:10.1016/0006-3223(94)91202-5

[10] Chen, J., Paredes, W., Van Praag, H.M., Lowinson, J.H. and Gardner, E.L. (1992) Presynaptic dopamine release is enhanced by 5-HT3 receptor activation in medial prefrontal cortex of freely moving rats. Synapse, 10, 264266. doi:10.1002/syn.890100308

[11] El Yacoubi, M., Costentin, J. and Vaugeois, J.M. (2003) Adenosine A2A receptors and depression. Neurology, 61, S82-S87. doi:10.1212/01.WNL.0000095220.87550.F6

[12] Braak, H., Ghebremedhin, E., Rüb, U., Bratzke, H. and Del Tredici, K. (2004) Stages in the development of Parkinson's disease-related pathology. Cell and Tissue Research, 318, 121-134. doi:10.1007/s00441-004-0956-9

[13] Paulus, W. and Jellinger, K. (1991) The neuropathologic basis of different clinical subgroups of Parkinson's disease. Journal of Neuropathology \& Experimental Neurology, 50, 743-755. doi:10.1097/00005072-199111000-00006

[14] Van Der Kooy, D. and Attori, T. (1980) Dorsal raph'e cells with collateral projections to the caudate-putamen and substantia nigra: A fluorescent retrograde double labeling study in the rat. Brain Research, 186, 1-7. doi:10.1016/0006-8993(80)90250-4

[15] Steinbush, H.W.M. (1984) Serotonin-immunoreactive neurons and their projections in the CNS. Handbook of Chemical Neuroanatomy: Classical Transmitter Receptors in the CNS, Part II, Elsevier, Amsterdam, 68-125.

[16] Herv'e, D., Pickel, V.M., Joh, T.H. and Beaudet, A. (1987) Serotonin axon terminals in the ventral tegmental area of the rat: Fine structure and synaptic input to dopaminergic neurons. Brain Research, 435, 71-83. doi:10.1016/0006-8993(87)91588-5

[17] Van Bockstaele, E.J., Biswas, A. and Pickel, V.M. (1993) Topography of serotonin neurons in the dorsal raph'e nucleus that send axon collaterals to the rat prefrontal cortex and nucleus accumbens. Brain Research, 624, 188-198. doi:10.1016/0006-8993(93)90077-Z

[18] Van Bockstaele, E.J., Cestari, D.M. and Pickel, V.M. (1994) Synaptic structure and connectivity of serotonin terminals in the ventral tegmental area: Potential sites for modulation of mesolimbic dopamine neurons. Brain Research, 647, 307-322.

doi:10.1016/0006-8993(94)91330-7

[19] Moukhles, H., Bosler, O., Bolam, J.P., Vall 'ee, A., Umbriaco, D., Geffard, M. and Doucet, G. (1997) Quantitative and morphometric data indicate precise cellular interactions between serotonin terminals and postsynaptic targets in rat substantia nigra. Neuroscience, 76, 11591171. doi:10.1016/S0306-4522(96)00452-6

[20] Schwarting, R.K. and Hustonn, J.P. (1996) Unilateral 6hydroxydopamine lesions of meso-striatal dopamine neurons and their physiological sequelae. Progress in Neurobiology, 49, 215-266. doi:10.1016/S0301-0082(96)00015-9

[21] Schwarting, R.K. and Huston, J.P. (1996) The unilateral 6-hydroxydopamine lesion model in behavioral brain research. Analysis of functional deficits, recovery and treat- ments. Progress in Neurobiology, 50, 275-331. doi:10.1016/S0301-0082(96)00040-8

[22] Deumens, R., Blokland, A. and Prickaerts, J. (2002) Modeling Parkinson's disease in rats: An evaluation of 6OHDA lesions of the nigrostriatal pathway. Experimental Neurology, 175, 303-317. doi:10.1006/exnr.2002.7891

[23] Schultz, W. (1982) Depletion of dopamine in the striatum as an experimental model of Parkinsonism: Direct effects and adaptive mechanisms. Progress in Neurobiology, 18, 121-166. doi:10.1016/0301-0082(82)90015-6

[24] Zigmond, M.J., Abercrombie, E.D., Berger, T.W., Grace, A.A. and Stricker, E.M. (1990) Compensations after lesions of central dopaminergic neurons: Some clinical and basic implications. Trends in Neurosciences, 13, 290-296. doi:10.1016/0166-2236(90)90112-N

[25] Forno, L.S. (1969) Concentric hyalin intraneuronal inclusions of Lewy type in the brain of elderly persons (50 incidental cases): Relationship to parkinsonism. Journal of the American Geriatrics Society, 17, 557-575.

[26] Koller, W.C. (1992) When does Parkinson's disease begin? Neurology, 42, 27-31.

[27] Sawle, G.V. (1993) The detection of preclinical Parkinson's Disease: What is the role of positron emission tomography? Movement Disorders, 8, 271-277. doi:10.1002/mds.870080304

[28] Rajput, A.H. (1994) Clinical features and natural history of Parkinson's disease (special consideration of aging). In: Calne, D.P., Ed., Neurodegenerative Diseases, Saunders, Philadelphia, 555-571.

[29] Poewe, W.H. and Wenning, G.K. (1998) The natural history of Parkinson's disease. Annals of Neurology, 44, 1-9.

[30] Gelb, D.J., Oliver, E. and Gilman, S. (1999) Diagnostic criteria for Parkinson's disease. Archives of Neurology, 56, 33-39. doi:10.1001/archneur.56.1.33

[31] Wolters, E.C., Francot, C., Bergmans, P., Winogrodzka, A., Booij, J., Berendse, H.W. and Stoof, J.C. (2000) Preclinical (premotor) Parkinson's disease. Journal of Neurology, 247, 103-109.

[32] Del Tredici, K., Rüb, U., de Vos, R.A.I., Bohl, J.R.E. and Braak, H. (2002) Where does Parkinson disease pathology begin in the brain? Journal of Neuropathology \& Experimental Neurology, 61, 413-426.

[33] Braak, H., Del Tredici, K., Rüb, U., de Vos, R.A.I., Jansen Steur, E.N.H. and Braak, E. (2003) Staging of brain pathology related to sporadic Parkinson's disease. Neurobiology of Aging, 24, 197-211. doi:10.1016/S0197-4580(02)00065-9

[34] Hawkes, C.H., Del Tredici, K. and Braak, H. (2007) Parkinson's disease: A dual-hit hypothesis. Neuropathology and Applied Neurobiology, 33, 599-614. doi:10.1111/j.1365-2990.2007.00874.x

[35] Cryan, J.F. and Holmes, A. (2005) The ascent of mouse: Advances in modelling human depression and anxiety. Nature Reviews, 4, 775-790. doi:10.1038/nrd1825

[36] Ramos, A., Pereira, E., Martins, G.C., Wehrmeister, T.D. and Izidio, G.S. (2008) Integrating the open field, elevated plus maze and light/dark box to assess different types of emotional behaviors in one single trial. Behav- 
ioural Brain Research, 193, 277-288. doi:10.1016/j.bbr.2008.06.007

[37] Lee, C.S., Sauer, H. and Bjorklund, A. (1996) Dopaminergic neuronal degeneration and motor impairments following axon terminal lesion by instrastriatal 6-hydroxydopamine in the rat. Neuroscience, 72, 641-653. doi:10.1016/0306-4522(95)00571-4

[38] Tadaiesky, M.T., et al. (2008) Emotional, cognitive and neurochemical alterations in a premotor stage model of Parkinson's disease. Neuroscience, 156, 830-840. doi:10.1016/j.neuroscience.2008.08.035

[39] Branchi, I., et al. (2008) Nonmotor symptoms in Parkinson's disease: Investigating early-phase onset of behavioral dysfunction in the 6-hydroxydopamine-lesioned rat model. Journal of Neuroscience Research, 86, 2050-2061. doi:10.1002/jnr.21642

[40] Sourani, D., Eitan, R., Gordon, N. and Goelman, G. (2012) The habenula couples the dopaminergic and the serotonergic systems: Application to depression in Parkinson's disease. European Journal of Neuroscience, 36, 28222829. doi:10.1111/j.1460-9568.2012.08200.x

[41] Winter, C., von Rumohr, A., Mundt, A., Petrus, D., Klein, J., Lee, T., Morgenstern, R., Kupsch, A. and Juckel, G. (2007) Lesions of dopaminergic neurons in the substantia nigra pars compacta and in the ventral tegmental area enhance depressive-like behavior in rats. Behavioral Brain Research, 184, 133-141. doi:10.1016/j.bbr.2007.07.002

[42] Santiago, R.M., Barbieiro, J., Lima, M.M., Dombrowski, P.A., Andreatini, R. and Vital, M.A. (2010) Depressive-like behaviors alterations induced by intranigral MPTP, 6-OHDA, LPS and rotenone models of Parkinson's Disease are predominantly associated with serotonin and dopamine. Progress in Neuro-Psychopharmacology \& Biological Psychiatry, 34, 1104-1114. doi:10.1016/j.pnpbp.2010.06.004

[43] Wang, S., et al. (2009) Unilateral lesion of the nigrostriatal pathway induces an increase of neuronal firing of the midbrain raphe nuclei 5-HT neurons and a decrease of their response to 5-HT(1A) receptor stimulation in the rat. Neuroscience, 159, 850-861.

doi:10.1016/j.neuroscience.2008.12.051

[44] Kaya, A.H., et al. (2008) Increased electrical and metabolic activity in the dorsal raphe nucleus of Parkinsonian rats. Brain Research, 1221, 93-97. doi:10.1016/j.brainres.2008.05.019

[45] Temel, Y., et al. (2007) Inhibition of 5-HT neuron activity and induction of depressive-like behavior by high-frequency stimulation of the subthalamic nucleus. Proceedings of the National Academy of Sciences of the United States of America, 104, 17087-17092. doi:10.1073/pnas.0704144104

[46] Pelled, G., Bergman, H., Ben-Hur, T. and Goelman, G. (2007) Manganese-enhanced MRI in a rat model of Parkinson's disease. Journal of Magnetic Resonance Imaging, 26, 863-870. doi:10.1002/jmri.21051

[47] Pautler, R.G., Silva, A.C. and Koretsky, A.P. (1998) In vivo neuronal tract tracing using manganese-enhanced magnetic resonance imaging. Magnetic Resonance in Medicine, 40, 740-748. doi:10.1002/mrm.1910400515
[48] Koretsky, A.P. and Silva, A.C. (2004) Manganese-enhanced magnetic resonance imaging (MEMRI). NMR in Biomedicine, 17, 529-539. doi:10.1002/nbm.940

[49] Saleem, K.S., et al. (2002) Magnetic resonance imaging of neuronal connections in the macaque monkey. Neuron, 34, 685-700. doi:10.1016/S0896-6273(02)00718-3

[50] Herkenham, M. and Nauta, W.J. (1977) Afferent connections of the habenular nuclei in the rat. A horseradish peroxidase study, with a note on the fiber-of-passage problem. The Journal of Comparative Neurology, 173 , 123-146. doi:10.1002/cne.901730107

[51] Geisler, S. and Trimble, M. (2008) The lateral habenula: No longer neglected. CNS Spectrums, 13, 484-489.

[52] Araki, M., McGeer, P.L. and Kimura, H. (1988) The efferent projections of the rat lateral habenular nucleus revealed by the PHA-L anterograde tracing method. Brain Research, 441, 319-330. doi:10.1016/0006-8993(88)91410-2

[53] Sartorius, A. and Henn, F.A. (2007) Deep brain stimulation of the lateral habenula in treatment resistant major depression. Medical Hypotheses, 69, 1305-1308. doi:10.1016/j.mehy.2007.03.021

[54] Sartorius, A., et al. (2010) Remission of major depression under deep brain stimulation of the lateral habenula in a therapy-refractory patient. Biological Psychiatry, 67, e9e11. doi:10.1016/j.biopsych.2009.08.027

[55] Morris, J.S., Smith, K.A., Cowen, P.J., Friston, K.J. and Dolan, R.J. (1999) Covariation of activity in habenula and dorsal raphe nuclei following tryptophan depletion. Neuroimage, 10, 163-172. doi:10.1006/nimg.1999.0455

[56] Smith, K.A., Morris, J.S., Friston, K.J., Cowen, P.J. and Dolan, R.J. (1999) Brain mechanisms associated with depressive relapse and associated cognitive impairment following acute tryptophan depletion. The British Journal of Psychiatry, 174, 525-529. doi:10.1192/bjp.174.6.525

[57] Caldecott-Hazard, S., Mazziotta, J. and Phelps, M. (1988) Cerebral correlates of depressed behavior in rats, visualized using 14C-2-deoxyglucose autoradiography. The Journal of Neuroscience, 8, 1951-1961.

[58] Shumake, J., Edwards, E. and Gonzalez-Lima, F. (2003) Opposite metabolic changes in the habenula and ventral tegmental area of a genetic model of helpless behavior. Brain Research, 963, 274-281. doi:10.1016/S0006-8993(02)04048-9

[59] Matsumoto, M. and Hikosaka, O. (2007) Lateral habenula as a source of negative reward signals in dopamine neurons. Nature, 447, 1111-1115. doi:10.1038/nature05860

[60] Ferraro, G., Montalbano, M.E., Sardo, P. and La Grutta, V. (1997) Lateral habenula and hippocampus: a complex interaction raphe cells-mediated. Journal of Neural Transmission, 104, 615-631. doi:10.1007/BF01291880

[61] Li, A., et al., (2006) Apomorphine-induced activation of dopamine receptors modulates FGF-2 expression in astrocytic cultures and promotes survival of dopaminergic neurons. FASEB Journal, 20, 1263-1265. doi:10.1096/fj.05-5510fje

[62] Tien, L.T., et al. (2003) Increased dopamine D2 receptor binding and enhanced apomorphine-induced locomotor 
activity in mu-opioid receptor knockout mice. Brain Research Bulletin, 61, 109-115.

doi:10.1016/S0361-9230(03)00077-7

[63] McCulloch, J., Savaki, H.E. and Sokoloff, L. (1980) In- fluence of dopaminergic systems on the lateral habenular nucleus of the rat. Brain Research, 194, 117-124.

$\underline{\text { doi:10.1016/0006-8993(80)91322-0 }}$ 